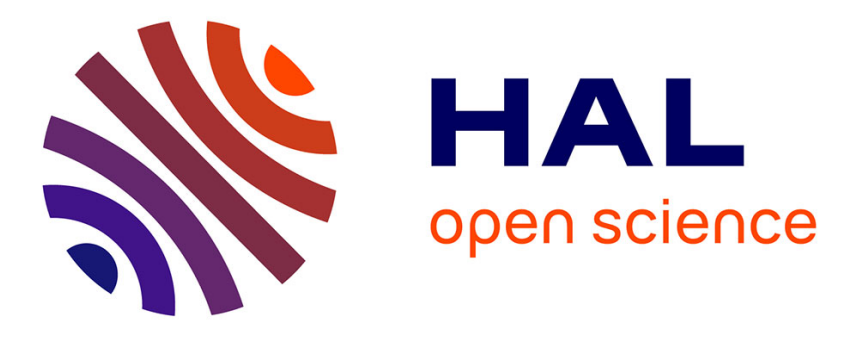

\title{
Extension of the Form-Closure Property to Underactuated Hands
}

Sébastien Krut, Vincent Begoc, Etienne Dombre, François Pierrot

\section{To cite this version:}

Sébastien Krut, Vincent Begoc, Etienne Dombre, François Pierrot. Extension of the Form-Closure Property to Underactuated Hands. IEEE Transactions on Robotics, 2010, 26 (5), pp.853-866. 10.1109/TRO.2010.2060830 . lirmm-00529721

\section{HAL Id: lirmm-00529721 https://hal-lirmm.ccsd.cnrs.fr/lirmm-00529721}

Submitted on 26 Oct 2010

HAL is a multi-disciplinary open access archive for the deposit and dissemination of scientific research documents, whether they are published or not. The documents may come from teaching and research institutions in France or abroad, or from public or private research centers.
L'archive ouverte pluridisciplinaire HAL, est destinée au dépôt et à la diffusion de documents scientifiques de niveau recherche, publiés ou non, émanant des établissements d'enseignement et de recherche français ou étrangers, des laboratoires publics ou privés. 


\title{
Extension of the Form-Closure Property to Underactuated Hands
}

\author{
Sébastien Krut, Vincent Bégoc, Etienne Dombre and François Pierrot
}

\begin{abstract}
The property of form-closure of a grasp, as generally defined in the literature, is based on the assumption that contact points between the hand and the object are fixed in space. However, this assumption is false when considering a grasp exerted by an underactuated hand, since in this case, it is not possible to control the position of each phalanx independently. In spite of researchers' interest in studying formclosure, none of the available published work on this subject takes into consideration the particular kinematics of underactuated hands. Actually, there are few available tools to qualify or quantify the stability of a grasp exerted by an underactuated hand, thus the design of underactuated hands mostly results from an intuitive approach. This paper aims to reduce this gap.

A classification of underactuated hands is proposed, based on the expression of contact forces. This highlights the influence of non-backdrivable mechanisms introduced in the transmission of the closing motion of the hand on the stability of the grasp. After demonstrating that the original definition of form-closure is not suitable for the underactuated grasps, a more general definition is formulated, which checks the stability of the assembly "object + hand". Using this new definition, a geometric method is proposed for the analysis of $1^{\text {st }}$ order form-closure of an underactuated grasp, as well as a simple rule for designing a hand capable of achieving $1^{\text {st }}$ order form-closed grasps. Finally, a method is proposed for the analysis of higher order form-closure of an underactuated grasp, based on a $1^{\text {st }}$ order model of the grasp.
\end{abstract}

Index Terms - Form-closure, Underactuated hands, Nonbackdrivability.

\section{INTRODUCTION}

$\mathrm{T}$ HE concept of underactuation makes it possible to create grippers which automatically adapt to the geometry of the grasped object, without requiring a large number of sensors or actuators, nor a complex control strategy. Thus, using a simple binary control, such as the one usually used to drive the closing/opening motion of a parallel-jaw gripper, an underactuated gripper permits to increase the number of

Manuscript received October 16, 2009.

S. Krut, E. Dombre and F. Pierrot are with the Montpellier Laboratory of Informatics, Robotics, and Microelectronics (LIRMM in French), a crossfaculty research entity of the Montpellier University of Sciences (UM2) and the French National Center for Scientific Research (CNRS) - 161 rue Ada, 34392 Montpellier Cedex 5, France. (Corresponding author: Sébastien Krut; phone/fax: +33467418588; e-mail: krut@lirmm.fr).

$\mathrm{V}$. Bégoc is with $\mathrm{B}+$ Equipment, a company specialized in research and development of automated systems and robotics - Avenue du Pic de Bertagne, 20 chemin de Saint Martin, 13420 Gemenos, France. contact points, resulting in an enveloping grasp that should be, a priori, more robust than a two-contact grasp. However, underactuated grippers are rarely used in the industry, first because most of gripping operations can be achieved using simple grippers dedicated to unique objects, but also because, in certain cases, underactuated grippers exhibit aberrant behavior which leads to an unstable grasp.

In [1], a characteristic phenomenon of underactuated hands, the ejection phenomenon, is described. In certain configurations of the finger, the distribution of contact forces degenerates, i.e. some phalanxes must exert a negative force on the object in order to guarantee static equilibrium of the finger. Since a contact action is unidirectional, equilibrium cannot be attained and the ejection of the object from the hand is initiated. The ejection phenomenon highlights the need for a more in-depth investigation of the stability of a grasp exerted by an underactuated hand, considering the particular kinematics of this type of mechanism. Indeed, there has been little work dedicated to studying the stability of a grasp exerted by an underactuated hand. There exist then only few tools for designing such an underactuated hand in order to maximize its ability to stabilize an object. As a result, its design often results from an intuitive approach.

There are two main criteria which can be used to characterize the robustness of a grasp: form-closure and force-closure. These two properties permit to perform a local and static study of the stability of a grasp. Form-closure describes the capability of a hand to prevent any motion of a grasped object. Force-closure describes the capability of a hand to counterbalance any external disturbances exerted on a grasped object, by applying a combination of contact forces which respect the capabilities of actuators and the conditions of friction [2]. In contrast to force-closure, form-closure is a purely geometric property since it does not depend on actuators capability nor on the eventual presence of frictional forces between the gripper and the object.

These two properties have been the subject of many investigations but, to the best of our knowledge, these have never been extended to the particular case of underactuated grasps $^{1}$ (even work in [3] on the analysis of underactuated grasps does not deal with the particular kinematics of underactuated hands). This paper focuses on form-closure and aims to extend this property to the case of underactuated grasps.

\footnotetext{
${ }^{1}$ As an abuse of language, a grasp realized by an underactuated mechanism is referred to as an underactuated grasp.
} 
II. STATE OF THE ART:

UNDERACTUATION AND FORM-CLOSURE

\section{A. Underactuation}

\section{Definition}

A mechanism is said to be underactuated, when it has fewer actuators than degrees of freedom [4].

An actuator is a sub-assembly which causes relative motion between the parts to which it is attached in response to a signal [5]. The number of degrees of freedom (dof) of a system is defined by the number of independent generalized coordinates required to define completely the configuration of a system at any instant of time [5]. Hence, determining the number of dof of a hand requires consideration of the coupling that is often introduced between the rotations of interphalanx joints. This is the case for several bionic hands, such as the DLR hands [6], the Robonaut's hand [7], the NAIST hand [8] and the hand of humanoid robot HRP-3 [9].

The Grübler-Kutzbach equation cannot be applied straightforwardly to determine the degree of freedom of an underactuated hand [10]. In fact, this method involves determining the order of a system of kinematic linear equations and it is therefore not applicable to underactuated hands which:

- use non-backdrivable mechanisms or anti-return mechanisms in the transmission of the opening/closing motion of the fingers, because they introduce non-linear constraints. This is the case for many underactuated hands such as the Barrett Hand [11] and the SARAH Hand [12],

- use compliant bodies to transfer the motor's torque to the fingers as is the case of the RTR II hand [13].

\section{Classification of underactuated hands}

There is a large diversity of mechanical devices which enable a hand to adapt to the geometry of an object. In [10], the author proposes to classify underactuated hands according to the type of mechanism used to realize the underactuation. In this paper, a classification is proposed which takes into account the characteristics of the grasp realized by the underactuated hand. This classification is based on the expression of contact forces as a function of the torques exerted by the actuators on the mechanism. Hence, an underactuated hand can be:

- (i) differential,

- (ii) compliant,

- (iii) self-locking,

- or have a combination of these three properties.

The first two categories can be characterized by the formulation given in [10].

(i) A mechanism is said to be differential when its behavior, from a kinetostatic point of view, can be described by the following equations:

$$
\begin{gathered}
F_{a}=F_{1}^{a} \cdot r_{1}=\cdots=F_{n}^{a} \cdot r_{n} \\
\dot{\theta}_{a}+\sum_{i=1}^{n} \frac{\dot{\theta}_{i}^{a}}{r_{i}^{a}}=0
\end{gathered}
$$

where $F_{a}$ is the actuation force, or the actuation torque, exerted on the input of the differential mechanism, and $F_{i}^{a}$ is the force or torque transmitted to the output $i$. $\left(\theta_{a}, \theta_{1}^{a}, \cdots, \theta_{n}^{a}\right)$ describes the configuration of the differential mechanism, $\left(\dot{\theta}_{a}, \dot{\theta}_{1}^{a}, \cdots, \dot{\theta}_{n}^{a}\right)$ the joint velocities and $\left(r_{1}^{a}, \cdots, r_{n}^{a}\right)$ the transmission ratios (that can depend on the configuration of the hand). A review of the state of the art of differential mechanisms can be found in [14].

(ii) A mechanism is said to be compliant when the forces exerted on its output can be written as a function of the configuration of the mechanism and the stiffness of the mechanical elements introduced into the transmission of the closing motion of the hand:

$$
\left[\begin{array}{llll}
F_{a} & F_{1}^{a} & \cdots & F_{n}^{a}
\end{array}\right]^{T}=\boldsymbol{R}\left[\begin{array}{llll}
\theta_{a} & \theta_{1}^{a} & \cdots & \theta_{n}^{a}
\end{array}\right]^{T}
$$

where $\boldsymbol{R}$ is the stiffness matrix of the mechanism.

(iii) The category of self-locking underactuated mechanisms is introduced in order to distinguish them from hands which have non-backdrivable or anti-return mechanisms in the transmission of the fingers' closing motion. Indeed, this has a strong influence on the expression of contact forces and, as a consequence, on the characteristics of the grasp since this type of mechanism prevents all return motion of the fingers or phalanxes whatever the disturbances exerted on the grasped object. Theoretically, the contact forces could then be infinite. Mathematically, a mechanism is said to be self-locking, when the configuration of the mechanism is constrained by a set of inequalities of the type:

$$
\delta_{i} \cdot \dot{\theta}_{i}^{a} \geq 0, \forall i=\{1, \cdots, n\}
$$

$\delta_{i}= \pm 1$ depending on the case.

Table I lists the main characteristics of various existing underactuated hands. For each hand, the table lists the number of dof, the number of actuators used to drive the closing/opening motion of the hand and the number of actuators used to modify the configuration of the hand. The type of underactuation used for the finger inputs and the phalanx inputs is listed separately, often being of different types. Finally, the number and type of the non-backdrivable mechanisms introduced in the mechanical transmission of the closing motion of the hand are indicated.

\section{B. State of the art for form-closure}

Form-closure can be described as the capability of a hand, or more generally of a set of contact constraints, to prevent motion of a grasped object. The following definition for formclosure is largely accepted in the literature:

A grasp is said to be form-closed if and only if for every motion of the object, at least one contact constraint is violated [2].

The term "contact constraint" relates to the fact that the relative motion of two solid bodies in contact is constrained by the condition of non-interpenetration. This contact constraint 
TABLE I

CHARACTERISTICS OF UNDERACTUATED ROBOTIC HANDS.

THE SYMBOL Ø INDICATES THAT THERE IS NO UNDERACTUATION OR NO NON-BACKDRIVEABLE MECHANISMS DEPENDING ON THE COLUMN.

\begin{tabular}{|c|c|c|c|c|c|c|c|}
\hline Name & $\begin{array}{l}Z \\
0 \\
0 \\
0 \\
0 \\
0 \\
0\end{array}$ & 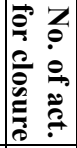 & 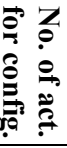 & $\begin{array}{l}\text { Type of underact. } \\
\text { between fingers }\end{array}$ & $\begin{array}{l}\text { Type of underact. } \\
\text { between phalanxes }\end{array}$ & $\begin{array}{c}\text { Non-backdriveable } \\
\text { mechanisms }\end{array}$ & Reference \\
\hline Soft Gripper & 18 & 2 & 0 & differential pulleys/cables & differential pulleys/cables & $\varnothing$ & {$[15]$} \\
\hline Hand of the LMS & 9 & 1 & 0 & differential with cables & differential pulleys/cables & $\varnothing$ & [16] \\
\hline TU Delft Hand & 5 & 1 & 0 & differential with cables & differential pulleys/cables & $\varnothing$ & {$[17]$} \\
\hline Barrett Hand & 7 & 3 & 1 & $\varnothing$ & self-locking & 6 wheels and worm drives & [11] \\
\hline TBM Prosthesis & 15 & 1 & 0 & compliant & differential four-bar & $\varnothing$ & {$[18]$} \\
\hline Southampton Hand Prosthesis & 15 & 4 & 2 & $\varnothing$ & differential four-bar & 6 wheels and worm drives & [19] \\
\hline Lopez Hand & 9 & 1 & 0 & self-locking & differential pulleys/cable & 9 bracing devices & {$[20]$} \\
\hline MARS & 12 & 3 & 3 & $\varnothing$ & differential four-bar & $\varnothing$ & {$[1]$} \\
\hline SARAH & 10 & 1 & 1 & self-locking & differential four-bar & 3 wheels and worm drives & {$[1]$} \\
\hline Laval Univ. Pneumatic Hand & 10 & 3 & 1 & $\varnothing$ & differential four-bar & 3 motorised anti-return valves & {$[12]$} \\
\hline RTR II & 9 & 1 & 1 & compliant & differential pulleys/cables & 1 wheel and worm drive & {$[13]$} \\
\hline SPRING Hand & 8 & 1 & 0 & compliant & differential pulleys/cables & 1 wheel and worm drive & {$[21]$} \\
\hline RTR IV & 14 & 1 & 0 & compliant & differential with cables & $\varnothing$ & {$[22]$} \\
\hline Nasser Prosthesis & 15 & 1 & 0 & self-locking & differential four-bar & 4 wheels and worm drives & {$[23]$} \\
\hline Cyberhand & 16 & 5 & 1 & $\varnothing$ & differential pulleys/cables & $\varnothing$ & {$[24]$} \\
\hline Laval Univ. Prosthesis & 17 & 1 & 0 & differential pulleys/cables & differential pulleys/cables & $\varnothing$ & {$[14]$} \\
\hline SDM Hand & 8 & 1 & 0 & differential pulleys/cables & differential with cables & $\varnothing$ & {$[25]$} \\
\hline iLimb & 11 & 5 & 1 & $\varnothing$ & differential with tendons & 5 wheels and worm drives & {$[26]$} \\
\hline Southampton End Effector Hand & 8 & 1 & 0 & differential four-bar & differential four-bar & $\varnothing$ & {$[27]$} \\
\hline
\end{tabular}

is generally approximated to $1^{\text {st }}$ order for sake of simplicity, and can be expressed by the following inequality [28]:

$$
f(\boldsymbol{\theta}, \dot{\boldsymbol{\theta}}, \boldsymbol{u}, \dot{\boldsymbol{u}}) \geq 0
$$

where $\boldsymbol{\theta}$ describes the configuration of the hand, $\dot{\boldsymbol{\theta}}$ is the vector of the joint velocities, $\boldsymbol{u}$ is the configuration (position and orientation) of the object in the operational space and $\dot{\boldsymbol{u}}$ is the vector of operational velocities (by abuse of notation, as $\dot{\boldsymbol{u}}$ might be different from $\frac{d u}{d t}$ depending on the parameterization for the orientation).

In the major part of the literature, the configuration of the hand is assumed to be fixed, which permits to avoid the kinematic study of the hand. This assumption implies that the control position of each contact phalanx is infinitely rigid and that motors are oversized in comparison with any disturbances likely to be exerted on the object. When approximating the contact constraint to $1^{\text {st }}$ order only, it can be written for the $i^{\text {th }}$ phalanx as following:

$$
\hat{\boldsymbol{n}}_{i}^{T} \dot{\boldsymbol{u}} \geq 0 \text { with } \hat{\boldsymbol{n}}_{i}=\left[\begin{array}{c}
\mathbf{I}_{3} \\
\boldsymbol{C}\left(\boldsymbol{p}_{i}\right)
\end{array}\right] \boldsymbol{n}_{i}
$$

where $\boldsymbol{C}\left(\boldsymbol{p}_{i}\right)$ is the cross product matrix associated with vector $\boldsymbol{p}_{i}, \boldsymbol{p}_{i}$ being the position vector of the $i^{\text {th }}$ contact point expressed in the reference frame attached to the palm of the hand $\mathfrak{R}_{p}$ and $\boldsymbol{n}_{i}$ being the normal vector at the $i^{\text {th }}$ contact point directed towards the grasped object, expressed in $\mathfrak{R}_{p}$.

Hence, it is possible to write the three following propositions [28]: (i) the grasp is not form-closed if there exists a motion of the object $\dot{\boldsymbol{u}} \in \mathbb{R}^{* d}$ such that all contact constraints are respected:

$$
\left\{\exists \dot{\boldsymbol{u}} \in \mathbb{R}^{* d}, \forall i \in\left\{1, \cdots, n_{c}\right\}, \hat{\boldsymbol{n}}_{i}^{T} \dot{\boldsymbol{u}}>0\right\}
$$

(ii) the grasp is $\mathbf{1}^{\text {st }}$ order form-closed if, and only if, for all motions of the object $\dot{\boldsymbol{u}} \in \mathbb{R}^{* d}$, at least one contact constraint is violated:

$$
\left\{\forall \dot{\boldsymbol{u}} \in \mathbb{R}^{* d}, \exists i \in\left\{1, \cdots, n_{c}\right\}, \hat{\boldsymbol{n}}_{i}^{T} \dot{\boldsymbol{u}}<0\right\}
$$

(iii) there is uncertainty on the form-closure of the grasp when condition $(i)$ is not met and there exists a motion of the object $\dot{\boldsymbol{u}} \in \mathbb{R}^{* d}$, such that the components of $\hat{\boldsymbol{n}}_{i}^{T} \dot{\boldsymbol{u}}$ are positive or zero:

$$
\left\{\exists \dot{\boldsymbol{u}} \in \mathbb{R}^{* d}, \forall i \in\left\{1, \cdots, n_{c}\right\}, \hat{\boldsymbol{n}}_{i}^{T} \dot{\boldsymbol{u}} \geq 0\right\}
$$

with $d$ the dimension of the configuration space of the object, and $n_{c}$ the number of contact points.

In cases (i) and (ii), the $1^{\text {st }}$ order approximation of the contact constraints is sufficient to conclude on the formclosure of the grasp. In case (iii), at least one component $\hat{\boldsymbol{n}}_{i}^{T} \dot{\boldsymbol{u}}$ is zero, so that the $1^{\text {st }}$ order approximation is not sufficient. It is then necessary to consider $2^{\text {nd }}$ or higher order effects, so as to be able to conclude on the form-closure of the grasp. If the conclusion is that form-closure is effectively achieved, one refers to $2^{\text {nd }}$ order (or higher order) form-closure.

In the following, the distinction is made between two definitions: $1^{\text {st }}$ order form-closure and $2^{\text {nd }}$ order form-closure 
[29]. Fig. 1 illustrates the inclusion of these different formclosure properties.

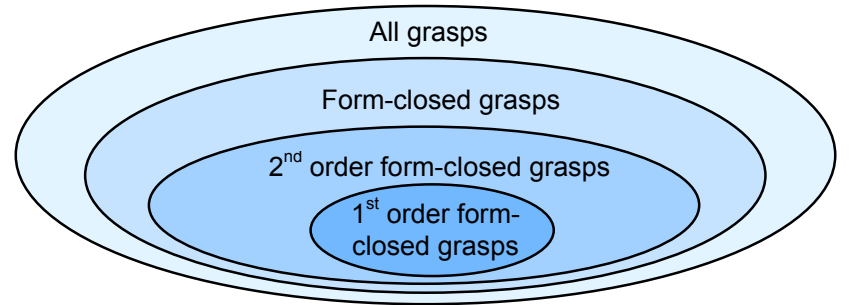

Fig. 1. Inclusion diagram of different properties of form-closure.

For example, the grasps represented in Fig. 2.a and Fig. 2.b are form-closed, whereas the grasp represented in Fig. 2.c is not. Grasp (a) is $1^{\text {st }}$ order form-closed, because a $1^{\text {st }}$ order modeling of the grasp is sufficient to demonstrate formclosure, whereas grasp (b) is $2^{\text {nd }}$ order form-closed, because $2^{\text {nd }}$ order modeling is required for demonstrating form-closure.

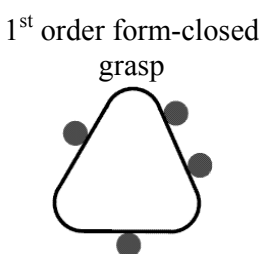

(a)

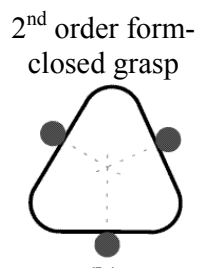

(b)

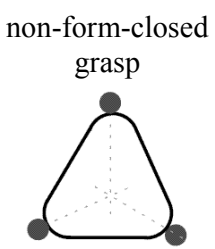

(c)
Fig. 2. Illustration of grasp types [30]. In all three situations, contact points prevent the object from translating. However, if they also prevent it from rotating in case (a), they do not in cases (b) and (c): the object is free to rotate around the point of concurrency of the contact normals. After an infinitesimal rotation, the object is stopped from rotating in case (b), whereas it is free to escape in case (c). This phenomenon can be modeled considering the local curvatures of the contact surfaces as explained in [31].

Several methods are available to analyze the form-closure of a grasp. In [32], the author proposed a geometric method in the object's plane, which is therefore applicable only in $2 \mathrm{D}$ cases. This method has been extended in [31] to the case of $2^{\text {nd }}$ order form-closed grasps, by considering the curvature of the contact surfaces. Another geometric method for $1^{\text {st }}$ order formclosure analysis was proposed in [32] and [29], which consists in verifying that the convex hull formed by the contact normal vectors (directed towards the object) and expressed in the operational space, contains the origin of the operational space in its interior. This convex hull is referred to as $\operatorname{conv}(\boldsymbol{P})$, where $\boldsymbol{P}$ is the projection matrix $\boldsymbol{P}=\left[\begin{array}{lll}\hat{\boldsymbol{n}}_{1} & \cdots & \hat{\boldsymbol{n}}_{n_{c}}\end{array}\right]^{T}$. The convex hull $\operatorname{conv}(\boldsymbol{P})$ corresponds to the convex hull of the set of points, $\mathcal{S}=\left\{\mathrm{P}_{1}, \cdots, \mathrm{P}_{n_{c}}\right\}$, where $\mathrm{P}_{i}$ is the point of coordinates $\hat{\boldsymbol{n}}_{i}\left(\hat{\boldsymbol{n}}_{i}^{T}\right.$ is the line $i$ of the matrix $\left.\boldsymbol{P}\right)$. The three propositions (7), (8), (9) can be rewritten using this new formalism as:

(i) the grasp is not form-closed if, and only if the origin of the operational space is strictly outside of the convex hull $\operatorname{conv}(\boldsymbol{P})$, (ii) the grasp is $\mathbf{1}^{\text {st }}$ order form-closed if the origin of the operational space is strictly inside the convex hull $\operatorname{conv}(\boldsymbol{P})$,

(iii) form-closure of the grasp is uncertain when the origin of the operational space is situated on the boundary of the convex hull $\operatorname{conv}(\boldsymbol{P})$.

There exists an analogous method, often used for forceclosure analysis, but applied in the dual space of operational forces. This consists in verifying that the convex hull, formed by the wrenches of the transmissible contact forces, contains the origin [34], [35]. When friction is negligible, these two methods are equivalent [29]. If a $2^{\text {nd }}$ order model of the grasp is available, then it is possible to apply the analysis method proposed by [29].

\section{A definition not suitable for underactuated grasps}

In this section, it is shown that the definition of formclosure, as it is presented in section B, does not apply to underactuated grasps. Therefore, four simple examples of twofinger grasps are studied (see Fig. 3). These grasps differ in terms of the type of actuation and transmission used to produce the closing motion of fingers. In order to simplify this analysis, in a way that will make it intuitive, an operational space of dimension one only is considered, i.e. only the translation of the object along $x$ is studied.

(a) The first mechanism is fully actuated (Fig. 3.a), i.e. both fingers are actuated independently by one motor. It is assumed that the position control of each jaw is infinitely rigid so that once in contact with the object, each jaw can be considered as fixed relative to the palm. In this case the object can move neither left nor right anymore; the grasp is form-closed.

(b) The second mechanism (Fig. 3.b) is underactuated by a "pulley/tendon" differential mechanism. The introduction of underactuation enables the two jaws to come in contact whatever the position of the object, using only one actuator. Intuition tells that, even if both jaws are in contact, the object is not immobilized and can move freely along $x$, relative to the frame. The grasp is not form-closed.

(c) The third mechanism is underactuated by a geared differential mechanism (Fig. 3.c). The closing motion of each jaw is transmitted via a "wheel and worm drive" nonbackdrivable mechanism. Once the two jaws are in contact, they cannot move back, meaning that the object is completely immobilized. The grasp is form-closed.

(d) The fourth mechanism is underactuated by a "pulley/tendon" differential mechanism. A single actuator drives the closing motion of the gripper by tightening cable 0 . This tension is transmitted to cables 1 and 2 which are connected to cable 0 at point A (Fig. 3.d). Once again, intuition tells that the object (in the position represented in the figure) tends to move towards the right, as cable 0 continues to be tightened. The grasp is not form-closed. Intuition lets foresee that, once re-centered, the object will no longer be able 
to move. It will be shown later in this paper, that, in this centered configuration, the grasp is $2^{\text {nd }}$ order form-closed.

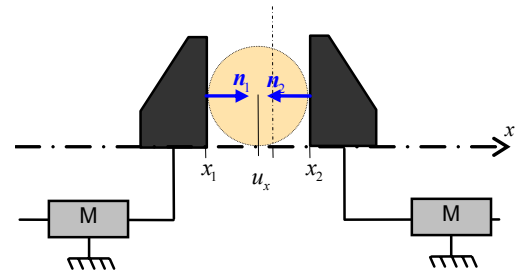

(a) A fully actuated form-closed grasp.

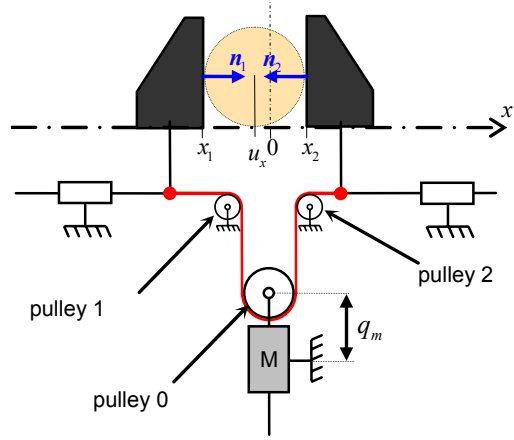

(b) An underactuated grasp, not form-closed because the object can move freely along $x$.

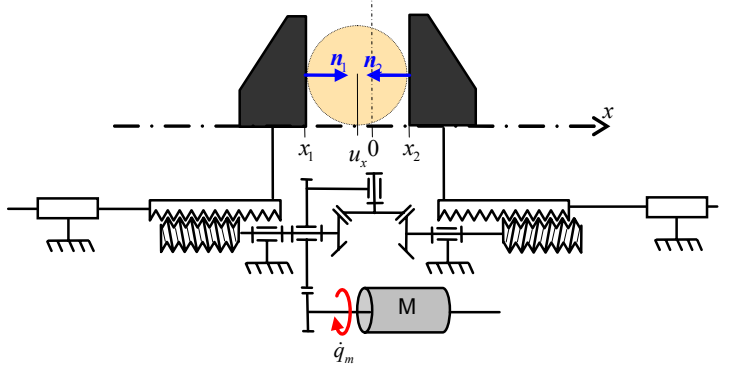

(c) An underactuated grasp, $1^{\text {st }}$ order form-closed thanks to the nonbackdrivable "wheel and worm drive" mechanisms

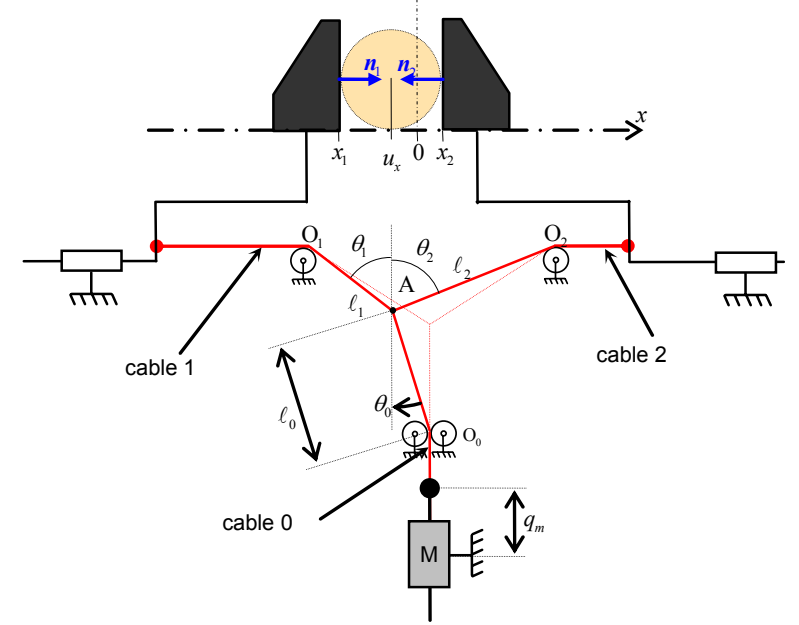

(d) An underactuated grasp, not form-closed in the position shown, because the object can move towards the right. The object tends to move towards the central position where the grasp attains $2^{\text {nd }}$ order form-closure.

Fig. 3. Diagram representing a two-contact grasp.
When applying directly the method of form-closure analysis discussed in section B, the conclusion is that all four grasps are form-closed. Indeed, when considering only the geometry of the contact points relatively to the object, these four grasps are identical. The convex hull $\operatorname{conv}(\boldsymbol{P})$ is reduced to the segment $[-1,1]$, with $\hat{n}_{1}=1$ and $\hat{n}_{2}=-1$, and it strictly contains the origin of the operational space in its interior.

As illustrated by the examples, this conclusion is false and the introduction of underactuation, or of non-backdrivable mechanisms, has a strong influence on the stability of the grasp (results are summarized in Table II). In the following section, a method is proposed for the analysis of form-closure for underactuated hands with several fingers and several phalanxes. This method considers the contact constraints and the constraints imposed by the non-backdrivable mechanisms.

TABLE II

SUMMARY OF THE DIFFERENT TYPES OF FORM-CLOSURE BEHAVIOUR OF THE UNDERACTUATED GRASPS DESCRIBED IN FIG. 3

\begin{tabular}{|c|c|c|c|c|}
\hline $\begin{array}{c}\text { Type of } \\
\text { actuation }\end{array}$ & $\begin{array}{c}\text { Fully act. } \\
\text { gripper (a) }\end{array}$ & $\begin{array}{c}\text { Underact. } \\
\text { gripper (b) }\end{array}$ & $\begin{array}{c}\text { Underact. } \\
\text { gripper (c) }\end{array}$ & $\begin{array}{c}\text { Underact. } \\
\text { gripper (d) }\end{array}$ \\
\hline $\begin{array}{c}\text { Capable of } \\
\text { form-closure }\end{array}$ & Yes & No & Yes & $\begin{array}{c}\text { Yes at one } \\
\text { position only }\end{array}$ \\
\hline $\begin{array}{c}\text { Type of form- } \\
\text { closure }\end{array}$ & $1^{\text {st }}$ order & $\varnothing$ & $1^{\text {st }}$ order & $2^{\text {nd }}$ order \\
\hline
\end{tabular}

\section{EXTENSION OF FORM-CLOSURE TO THE CASE OF} UNDERACTUATED GRASPS

\section{A. Proposal for a new definition}

In this section, the definition of form-closure is reformulated so that it applies to the case of underactuated grasps. Since immobility of contact points is not guaranteed, not only the motions of the object have to be studied but also the motions of the grasp, i.e. the motions of the overall system including the object and the hand. Thus, checking formclosure of an underactuated grasp would now require verifying that any variation in the configuration of the grasp is prevented by a set of unilateral kinematic constraints, i.e. the contact constraints and the constraints imposed by the nonbackdrivable mechanisms. Indeed, it has been shown in section II.C that the introduction of non-backdrivable mechanisms into the transmission of the closing motion of an underactuated gripper has a direct influence on the stability of the grasp. This explains why a large number of underactuated hands described in section II.A.2 use non-backdrivable mechanisms without, however, justifying it.

The definition of form-closure is then reformulated as follows:

A grasp is said to be form-closed if, and only if, for any variation of the configuration of the grasp at least one of the unilateral kinematic constraints is violated.

\section{B. Mathematical formulation}

The goal of this section is to determine a mathematical formulation of the constraints imposed by a non-backdrivable mechanism and to rewrite the kinematic contact constraints. 
For sake of simplicity, a $1^{\text {st }}$ order modeling of the grasp is considered. In the following, the mathematical formulations are given for enveloping grasps, i.e. hands that encircle the object in such a way that several phalanxes of all fingers are in contact with the object.

\section{Reformulation of the contact constraints}

Since phalanxes are not considered as being fixed in space, it is necessary to reformulate the non-interpenetration condition (6) by introducing the displacement velocities of the phalanxes. Let us consider the general case of an enveloping grasp exerted by a hand with $m$ fingers and $n$ phalanxes per finger (Fig. 4.). Each phalanx has a maximum of one contact point with the object. When all the phalanxes are in contact, the non-interpenetration conditions can be written as:

$$
v_{i, k}^{y, o} \geq v_{i, k}^{y, p}, \forall i=\{1, \ldots, n\} \text { et } \forall k=\{1, \ldots, m\}
$$

where $v_{i, k}^{y, o}$ and $v_{i, k}^{y, p}$ are the velocities of the contact point $\mathrm{A}_{i, k}$ belonging respectively to the object and the phalanx $i$ of finger $k$, along the normal of the contact $\boldsymbol{y}_{i, k}$.

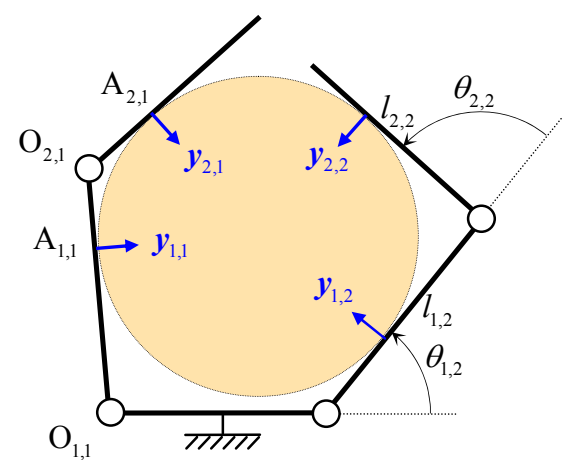

Fig. 4. Enveloping grasp of a disc by a hand with two fingers and two phalanxes per finger.

The conditions of non-interpenetration can be expressed in a matrix form using the Jacobian matrix of the finger $\boldsymbol{J}_{k}$, relating the vector $\dot{\boldsymbol{\theta}}_{k}$ to the vector $\boldsymbol{v}_{k}^{y, p}$. When making the assumption of friction free contacts, one obtains:

$$
\boldsymbol{J}_{k}=\left[\begin{array}{ccccc}
k_{1, k} & 0 & 0 & \cdots & 0 \\
\boldsymbol{r}_{12, k}^{T} \boldsymbol{x}_{2, k} & k_{2, k} & 0 & \cdots & 0 \\
\cdots & \cdots & \cdots & \cdots & \cdots \\
\boldsymbol{r}_{1 n, k}^{T} \boldsymbol{x}_{n, k} & \boldsymbol{r}_{2 n, k}^{T} \boldsymbol{x}_{n, k} & \boldsymbol{r}_{3 n, k}^{T} \boldsymbol{x}_{n, k} & \cdots & k_{n, k}
\end{array}\right]
$$

where $\boldsymbol{v}_{k}^{y, p}=\left[v_{1, k}^{y, p}, v_{2, k}^{y, p}, \cdots, v_{n, k}^{y, p}\right]^{T}$ is the vector of phalanx velocities of finger $k$ along the normals to the phalanxes and $\dot{\boldsymbol{\theta}}_{k}=\left[\dot{\theta}_{1, k}, \dot{\theta}_{2, k}, \cdots, \dot{\theta}_{n, k}\right]^{T}$ is the vector of joint velocities of finger $k$.

The terms of $\boldsymbol{J}_{k}$ are given by:

$$
\boldsymbol{r}_{i j, k}^{T} \boldsymbol{x}_{j, k}=k_{j, k}+\sum_{p=i}^{j-1} l_{p, k} \cos \left(\sum_{q=p+1}^{j} \theta_{q, k}\right), i<j
$$

$$
k_{i, k}=\boldsymbol{r}_{i i, k}^{T} \boldsymbol{x}_{i, k}
$$

The generalization to $m$ fingers of equation (11) is written using the Jacobian matrix of the hand, $\boldsymbol{J}$ :

$$
\begin{gathered}
\boldsymbol{v}^{y, p}=\boldsymbol{J} \dot{\boldsymbol{\theta}} \\
\boldsymbol{J}=\operatorname{diag}\left(\boldsymbol{J}_{1}, \cdots, \boldsymbol{J}_{m}\right)
\end{gathered}
$$

where $\boldsymbol{v}^{y, p}=\left[\boldsymbol{v}_{1}^{y, p T}, \boldsymbol{v}_{2}^{y, p T}, \cdots, \boldsymbol{v}_{m}^{y, p T}\right]^{T}$ is the vector of phalanx velocities of the hand and $\dot{\boldsymbol{\theta}}=\left[\dot{\boldsymbol{\theta}}_{1}^{T}, \dot{\boldsymbol{\theta}}_{2}^{T}, \cdots, \dot{\boldsymbol{\theta}}_{m}^{T}\right]^{T}$ is the vector of joint velocities of the hand.

In the same way, the linear relation between the vector of operational velocities of the object, $\dot{\boldsymbol{u}}$, and the vector $\boldsymbol{v}^{y, o}$, can be expressed using the projection matrix $\boldsymbol{P}$ :

$$
\begin{gathered}
\boldsymbol{v}^{y, o}=\boldsymbol{P} \dot{\boldsymbol{u}} \\
\boldsymbol{P}=\boldsymbol{N}^{T} \boldsymbol{G}^{T} \\
\boldsymbol{G}=\left[\begin{array}{ccc}
\mathbf{I}_{3} & \cdots & \mathbf{I}_{3} \\
\boldsymbol{C}\left(\boldsymbol{r}_{1}\right) & \cdots & \boldsymbol{C}\left(\boldsymbol{r}_{n_{c}}\right)
\end{array}\right] \\
\boldsymbol{N}=\operatorname{diag}\left(\boldsymbol{y}_{1}, \cdots, \boldsymbol{y}_{n_{c}}\right)
\end{gathered}
$$

$\boldsymbol{v}^{y, o}=\left[\boldsymbol{v}_{1}^{y, o T}, \boldsymbol{v}_{2}^{y, o T}, \cdots, \boldsymbol{v}_{m}^{y, o T}\right]^{T}$ is the vector of the velocities of the contact points attached to the object, projected on the normal vectors of the contact surfaces, and $\boldsymbol{C}\left(\boldsymbol{r}_{i}\right)$ represents the cross product matrix associated with vector $\boldsymbol{r}_{i}$.

Equation (10) can then be reformulated in the following form:

$$
\boldsymbol{v}^{y, o}-\boldsymbol{v}^{y, p} \geq 0
$$

Hence the $1^{\text {st }}$ order approximation of the noninterpenetration condition for an enveloping grasp can be written as:

$$
\boldsymbol{P} \dot{\boldsymbol{u}}-\boldsymbol{J} \dot{\boldsymbol{\theta}} \geq 0
$$

Relation (22) assumes that each phalanx is in contact with the object. When this is not the case, a selection matrix $\boldsymbol{S}$ is introduced:

$$
\boldsymbol{S}(\boldsymbol{P} \dot{\boldsymbol{u}}-\boldsymbol{J} \dot{\boldsymbol{\theta}}) \geq 0
$$

where $S$ is the identity matrix from which line $j$ is removed if phalanx $i$ of finger $k$ is not in contact with the object $(j=(k-1) n+i), n$ being the number of phalanxes.

\section{Constraints imposed by unidirectional mechanisms}

In section II.C, the influence of unidirectional mechanisms on form-closure of underactuated grasps was highlighted. In this section, we propose to model their action on the grasping mechanism in the form of a unilateral condition, as it has been done for contact constraints.

\section{Classification of unidirectional mechanisms}

A mechanism is said to provide unidirectional transmission of motion, when its output velocity is such that:

$$
\dot{q}_{s} \geq 0
$$


where $\dot{q}_{s}$ is the output velocity of the unidirectional mechanism.

In the following, the abbreviated version "unidirectional mechanism" is used to refer to this type of mechanism. Antireturn and non-backdrivable mechanisms can be included in this category.

Among the available anti-return mechanisms are:

- blocking mechanisms by obstacle, such as the "pawl and ratchet" mechanism (Fig. 5.a) or the sliding gear,

- locking mechanisms by bracing or wedging such as a freewheel (with ramp or cam) (Fig. 5.b), wrap spring couplings (Fig. 5.c) or sliding/bracing mechanisms, such as those used in the underactuated hand designed by [20],

- a non-return valve when fluid energy is used.

In the case of the free-wheel mechanism and wrap spring coupling, the unilateral condition is described differently:

$$
\dot{\theta}_{\text {out }} \geq \dot{\theta}_{\text {in }}
$$

where $\dot{\theta}_{\text {out }}$ and $\dot{\theta}_{\text {in }}$ are the output and input velocities of the mechanism respectively. This condition is identical to condition (24) when the input velocity is zero.

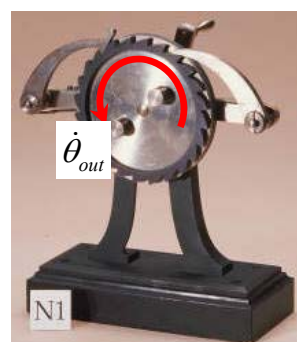

(a)

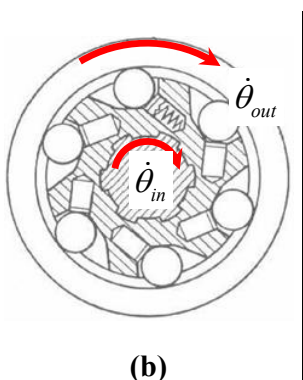

(b)

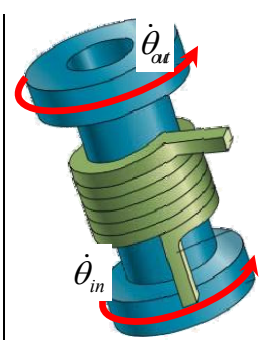

(c)
Fig. 5. Examples of anti-return mechanisms (a) "pawl and ratchet" mechanism (http://kmoddl.library.cornell.edu), (b) freewheel, (c) wrap spring coupling.

Non-backdrivable mechanisms can also be used leading to the unilateral condition (24). A mechanism is said to be nonbackdrivable when it is incapable of transmitting motion and power from the output to the input [5], as for instance:

- the triangular wedge,

- The "wheel and worm drive" mechanism (Fig. 6.a),

- The "rack and worm drive" mechanism,

- The "lead screw and nut" mechanism (Fig. 6.b).

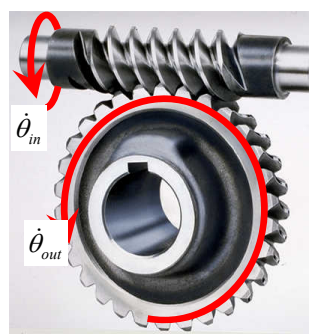

(a)

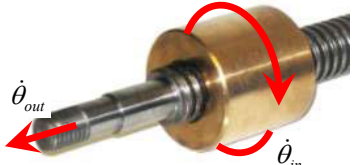

(b)
Fig. 6. Examples of non-backdrivable mechanisms: (a) "wheel and worm drive", (b) "lead screw and nut" mechanism.

A non-backdrivable mechanism operates differently from an anti-return mechanism. Nevertheless, it is possible to impose a unidirectional transmission of motion $\dot{q}_{s} \geq 0$ by maintaining a positive or null force on the input of the nonbackdrivable mechanism

In practice, it is noticeable that designers of underactuated hands prefer to introduce non-backdrivable mechanisms. The advantage of these mechanisms is that it is sufficient to invert the direction of rotation at the input in order to invert the sense of rotation at the output, and hence to enable opening of the gripper. In the case of a unidirectional mechanism, a mechanism is required to deactivate the anti-return function, as for instance the solution proposed in [20] to deactivate a sliding/bracing mechanism.

\section{Mathematical formulation}

This section aims to present a general method for expressing the constraints imposed by unidirectional mechanisms in a matrix form:

$$
\dot{\boldsymbol{q}}_{u}=\boldsymbol{K} \dot{\boldsymbol{\theta}} \geq 0
$$

where $\dot{\boldsymbol{q}}_{u}$ is the vector of output velocities of the unidirectional mechanisms and $\boldsymbol{K}$ is the matrix relating the vector of joint velocities of the hand $\dot{\boldsymbol{\theta}}$ to the vector $\dot{\boldsymbol{q}}_{u}$.

The expression of the constraint imposed by a unidirectional mechanism, as a function of the joint velocities of the hand, depends on the position of this mechanism in the transmission system of the closing motion. In the case of the Lopez hand [20], the anti-return mechanisms are positioned directly behind each phalanx and, for the SARAH hand [1], a "lead screw and nut" mechanism transmits the closing motion to each underactuated finger. It is therefore difficult to propose a general method for writing the kinematic constraints imposed by the unidirectional mechanisms. Nevertheless, it can be noted that there is a preference among designers to position a non-backdrivable mechanism on the input of each finger. This is the case for the MARS [1] and SARAH hands, for the Southampton hand [19], the TBM hand [18] (except for the thumb) and the Nasser hand [23].

Let us write the expression of matrix $\boldsymbol{K}$ for the SARAH hand. For each of the three fingers, a non-backdrivable "lead screw and nut" mechanism imposes the following inequality:

$$
\dot{\theta}_{a, k} \geq 0, \text { for } k=1, \cdots, 3
$$

where $\dot{\theta}_{a, k}$ is the velocity of the actuation bar $a_{1, k}$ of the finger $k$ (Fig. 7).

It can thus be written:

$$
\begin{gathered}
\dot{\boldsymbol{q}}_{u}=\left[\dot{\theta}_{a, 1}, \dot{\theta}_{a, 2}, \dot{\theta}_{a, 3}\right]^{T} \\
\dot{\boldsymbol{\theta}}=\left[\dot{\theta}_{1,1}, \dot{\theta}_{2,1}, \dot{\theta}_{3,1}, \dot{\theta}_{1,2}, \dot{\theta}_{2,2}, \dot{\theta}_{3,2}, \dot{\theta}_{1,3}, \dot{\theta}_{2,3}, \dot{\theta}_{3,3}\right]^{T}
\end{gathered}
$$

The method of kinetostatic analysis of underactuated fingers proposed by [36] allows us to write the relation between the joint velocities of the finger and the output velocities of the non-backdrivable mechanisms. Hence, again for the SARAH hand, the matrix $\boldsymbol{K}$ is written as: 


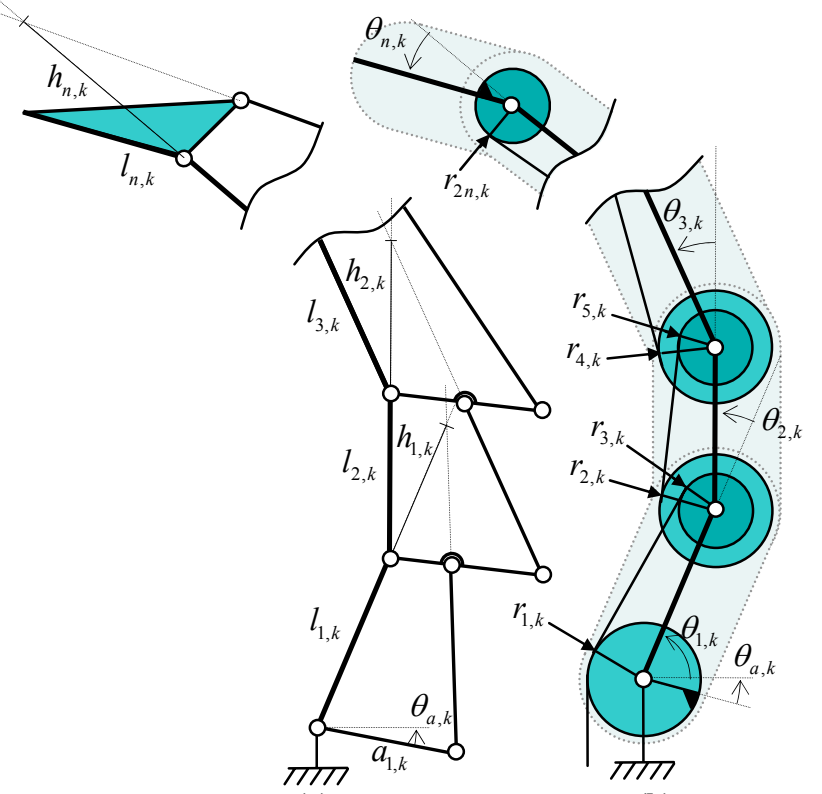

(a)

(b)

Fig. 7. Modeling of an underactuated finger with $\mathrm{n}$ phalanxes and with (a) a "4-bar" differential mechanism and (b) a "pulley/cable" differential mechanism.

$\boldsymbol{K}=\left[\begin{array}{ccccccccc}2-X_{1,1} & -X_{2,1} & -X_{3,1} & 0 & 0 & 0 & 0 & 0 & 0 \\ 0 & 0 & 0 & 2-X_{1,2} & -X_{2,2} & -X_{3,2} & 0 & 0 & 0 \\ 0 & 0 & 0 & 0 & 0 & 0 & 2-X_{1,3} & -X_{2,3} & -X_{3,3}\end{array}\right](30)$

For a "4-bar" underactuated mechanism, the expression of $X_{n, k}$ is:

$$
X_{n, k}=-\prod_{i=2}^{n} \frac{h_{i, k}}{h_{i, k}+l_{i-1, k}}
$$

The analytical expression for $h_{i, k}$ is given in [36].

For a "pulley/tendon" underactuated mechanism, the expression of $X_{n, k}$ is:

$$
X_{n, k}=-\prod_{i=1}^{n} \frac{r_{2 i, k}}{r_{2 i-1, k}}
$$

where $r_{i, k}$ represent the radius of the different pulleys (see Fig. 7.b).

\section{Kinematic modeling of the general case}

The aim of this section is to propose a global approach for studying $1^{\text {st }}$ order form-closure of a grasp. This approach allows to treat cases where the hands are or are not underactuated, where all the phalanxes are or are not in contact, and where the palm is or is not in contact with the object. Therefore, the vector of unilateral constraints $\dot{\boldsymbol{q}}_{c}$ is introduced, containing all the unilateral kinematic constraints of the problem. This vector is constructed in such a way that the $i^{\text {th }}$ component must be positive or zero, otherwise the corresponding constraint is violated. The linear relation between the vector of the motion of the grasp $\dot{\boldsymbol{w}}$ and the constraint vector $\dot{\boldsymbol{q}}_{c}$ can be written using the matrix $\boldsymbol{M}$ :

$$
\begin{gathered}
\dot{\boldsymbol{q}}_{c}=\boldsymbol{M} \dot{\boldsymbol{w}} \\
\dot{\boldsymbol{q}}_{c}=\left[\begin{array}{c:c}
\boldsymbol{S} \boldsymbol{P} & -\boldsymbol{S} \boldsymbol{J} \\
\mathbf{0} & \boldsymbol{K} \\
\hat{\boldsymbol{n}}_{p}^{T} & 0
\end{array}\right]\left[\begin{array}{c}
\dot{\boldsymbol{u}} \\
\dot{\boldsymbol{\theta}}
\end{array}\right]
\end{gathered}
$$

where $\dot{\boldsymbol{w}}=\left[\dot{\boldsymbol{u}}^{T}, \dot{\boldsymbol{\theta}}^{T}\right]^{T}$, by abuse of notation, represents the vector of infinitesimal displacements of the configuration of the grasp. This vector is the concatenation of the vector of operational velocities of the object $\dot{\boldsymbol{u}}$, with the vector of joint velocities of the hand $\dot{\boldsymbol{\theta}}$.

$$
\hat{\boldsymbol{n}}_{p}=\left[\begin{array}{c}
\mathbf{I}_{3} \\
\boldsymbol{C}\left(\boldsymbol{p}_{p}\right)
\end{array}\right] \boldsymbol{n}_{p}
$$

where $\boldsymbol{n}_{p}$ is the normal to the palm/object contact and $\boldsymbol{p}_{p}$ is the position of the palm/object contact, expressed in the hand reference frame.

The last line of matrix $\boldsymbol{M}$ is considered only when the palm is in contact with the object. A contact with the palm is treated differently because the palm can be considered fixed relatively to the hand reference frame. If the contact with the object is a line (respectively a surface), then it can be modeled by two (respectively three) contact points.

Hence, it is possible to rewrite propositions (i), (ii) and (iii) from section II.B, applied to the case of underactuated grasps, in the following form:

(i) the grasp is not form-closed if there exists a motion of the grasp $\dot{\boldsymbol{w}} \in \mathbb{R}^{* g}$ such that all components $\dot{q}_{c, i}$ are strictly positive:

$$
\left\{\exists \dot{\boldsymbol{w}} \in \mathbb{R}^{* g}, \forall i \in\left\{1, \cdots, n_{k}\right\}, \dot{q}_{c, i}>0\right\}
$$

(ii) the grasp is $\mathbf{1}^{\text {st }}$ order form-closed if, and only if, for all motions of the grasp $\dot{\boldsymbol{w}} \in \mathbb{R}^{* g}$, at least one of the unilateral constraints is violated:

$$
\left\{\forall \dot{\boldsymbol{w}} \in \mathbb{R}^{* g}, \exists i \in\left\{1, \cdots, n_{k}\right\}, \dot{q}_{c, i}<0\right\}
$$

(iii) there is uncertainty regarding the form-closure of the grasp when condition (i) is not met and there exists a motion of the grasp $\dot{\boldsymbol{w}} \in \mathbb{R}^{* g}$ such that components $\dot{q}_{c, i}$ are positive or zero:

$$
\left\{\exists \dot{\boldsymbol{w}} \in \mathbb{R}^{* g}, \forall i \in\left\{1, \cdots, n_{k}\right\}, \dot{q}_{c, i} \geq 0\right\}
$$

with the following notations:

- $g=n_{p}+d$ is the dimension of the grasp configuration space,

- $n_{p}$ is the dimension of the hand configuration space,

- $d$ is the dimension of the configuration space of the object,

- $n_{k}=n_{c}+n_{u}$ is the number of unilateral constraints of the problem,

- $n_{c}$ is the number of unilateral contact constraints,

- $n_{u}$ is the number of unidirectional mechanism constraints. 
Note that the original definition of $1^{\text {st }}$ order form-closure (8) is a necessary condition of the extended definition of $1^{\text {st }}$ order form-closure (37). Indeed, the domain of grasp motions $\dot{\boldsymbol{w}}$ such that $\dot{\boldsymbol{u}} \in \mathbb{R}^{* d}$ and $\dot{\boldsymbol{\theta}}=\mathbf{0}$ is a subset of the domain of grasp motions such that $\dot{\boldsymbol{w}} \in \mathbb{R}^{* g}$.

\section{Case study}

This new definition of form-closure can be illustrated by considering the two parallel-jaw grasps of Fig. 3.c and Fig. 3.d. Let us check that all grasp motions $\dot{\boldsymbol{w}}=\left[\begin{array}{lll}\dot{u}_{x} & \dot{x}_{1} & \dot{x}_{2}\end{array}\right]^{T}$ are prevented by the unilateral kinematic constraints, i.e. the contact constraints and those imposed by the unidirectional mechanisms. $\dot{u}_{x}$ represents an infinitesimal displacement of the object along $x$ and $\dot{x}_{1}$ and $\dot{x}_{2}$ are the infinitesimal displacements of jaws 1 and 2 respectively.

For the underactuated gripper represented in Fig. 3.c, the contact constraints are written as:

$$
\dot{x}_{1} \leq \dot{u}_{x} \leq \dot{x}_{2}
$$

The constraints imposed by the non-backdrivable mechanisms are written as:

$$
\dot{x}_{1} \geq 0 \text { and } \dot{x}_{2} \leq 0
$$

Rewriting all these unilateral constraints in a matrix form gives:

$$
\dot{\boldsymbol{q}}_{c}=\left[\begin{array}{ccc}
1 & -1 & 0 \\
-1 & 0 & 1 \\
0 & 1 & 0 \\
0 & 0 & -1
\end{array}\right] \dot{\boldsymbol{w}}
$$

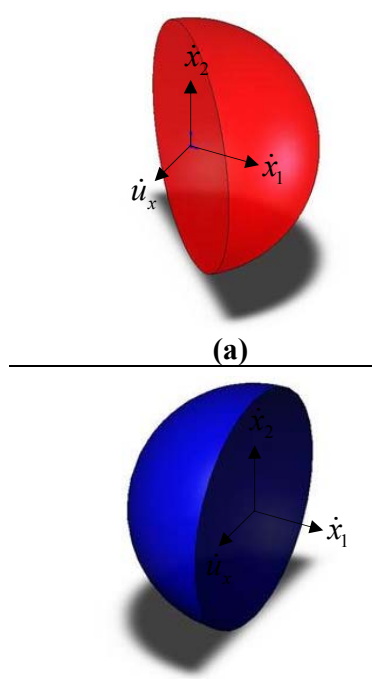

(c)

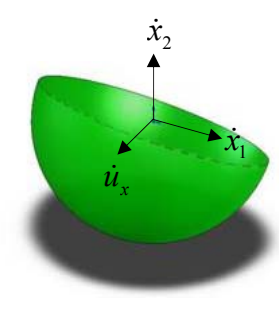

(b)

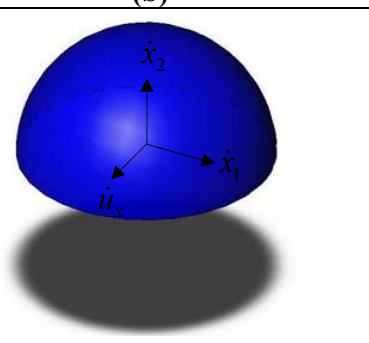

(d)
Fig. 8. Representation of the domains of grasp motions $\dot{\boldsymbol{w}}$ forbidden by the contact constraints (a) and (b), and by the two unilateral "wheel and worm drive" mechanisms (c) and (d), for the grasp shown in Fig. 3.c. The union of the forbidden domains covers the entire space of grasp motions except the null vector. The grasp is therefore $1^{\text {st }}$ order form-closed.

The domains of grasp motions $\dot{\boldsymbol{w}}$, which are forbidden by both contact constraints of left jaw/object and right jaw/object, are shown in Fig. 8.a and Fig. 8.b respectively. The domains of motion forbidden by the two non-backdrivable "wheel and worm drive" mechanisms are shown in Fig. 8.c and Fig. 8.d. The union of these four open domains covers the whole space of grasp motions except the null vector. The "object + hand" system is therefore immobilized: the grasp is $1^{\text {st }}$ order formclosed.

Let us now consider the underactuated parallel-jaw gripper shown in Fig. 3.d. The contact constraints are identical to the preceding case:

$$
\dot{x}_{1} \leq \dot{u}_{x} \leq \dot{x}_{2}
$$

It is assumed that the actuator imposes a unidirectional transmission of motion such that:

$$
\dot{q}_{m} \leq 0
$$

The underactuated mechanism imposes the following relationship:

$$
\dot{\ell}_{1} \sin \left(\theta_{2}-\theta_{0}\right)+\dot{\ell}_{2} \sin \left(\theta_{1}+\theta_{0}\right)+\dot{\ell}_{0} \sin \left(\theta_{1}+\theta_{2}\right)=0
$$

where $\ell_{i}$ is the distance from point $\mathrm{O}_{i}$ to point $\mathrm{A}$ and $\theta_{i}$ is the angle between cable $i$ and the vertical.

Equation (44) can be rewritten as follows:

$$
\dot{x}_{1} \sin \left(\theta_{2}-\theta_{0}\right)-\dot{x}_{2} \sin \left(\theta_{1}+\theta_{0}\right)+\dot{q}_{m} \sin \left(\theta_{1}+\theta_{2}\right)=0
$$

When the object is in a central position relatively to the gripper $\left(u_{x}=0\right)$, then $\theta_{0}=0$ and $\theta_{1}=\theta_{2}$. Relations (43) and (45) imposed by the differential mechanism when the object is in the central position, give:

$$
\dot{x}_{2}-\dot{x}_{1} \leq 0
$$

Rewriting all of these unilateral constraints in a matrix form gives:

$$
\dot{\boldsymbol{q}}_{c}=\left[\begin{array}{ccc}
1 & -1 & 0 \\
-1 & 0 & 1 \\
0 & 1 & -1
\end{array}\right] \dot{\boldsymbol{w}}
$$

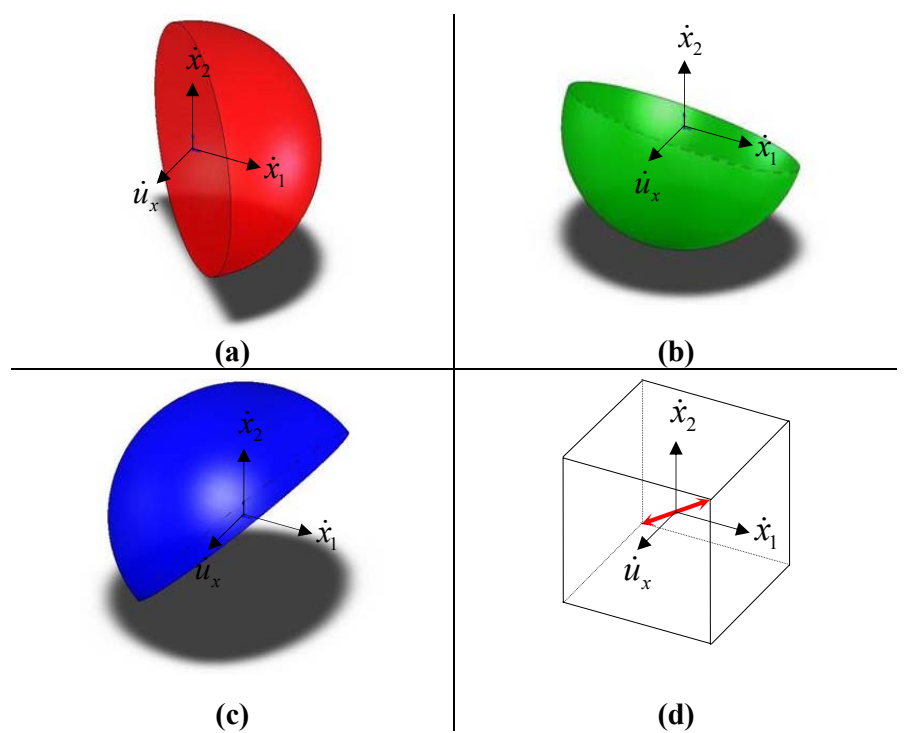

Fig. 9. Representation of the domains of motion $\dot{\boldsymbol{w}}$ forbidden by the contact constraints (a) and (b), and by the unidirectional actuator (c), for the grasp represented in Fig. 3.d when the object is in the centered position. The union of the three open domains is represented in (d). It is a line which corresponds to the direction of permitted motions; the grasp is not $1^{\text {st }}$ order form-closed. 
The domains of grasp motions $\dot{\boldsymbol{w}}$, forbidden by the contact constraints, are represented in Fig. 9.a and Fig. 9.b. The domain of grasp motions forbidden by the actuator is shown in Fig. 9.c. The union of the three open domains does not cover all of the motion space of the grasp, since the motions in the direction determined by the equality $\dot{u}_{x}=\dot{x}_{1}=\dot{x}_{2}$ are permitted (Fig. 9.d). The grasp is therefore not $1^{\text {st }}$ order formclosed.

It is interesting to note that the matrix of equation (47) is the same as that which can be used to model the gripper of Fig. 3.b. The results obtained for the gripper of Fig. 3.d therefore remain valid for that of Fig. 3.b: this gripper does not produce a $1^{\text {st }}$ order form-closed grasp. It will be shown how these two grippers differ in the last section of the paper: gripper (d) is able to produce a higher order form-closed grasp whereas gripper (b) cannot produce any form-closed grasp.

\section{EXTENSION OF PREVIOUS WORKS FROM THE LITERATURE TO UNDERACTUATED GRASPS}

The formalism adopted to define form-closure, extended to the case of underactuated grasps, is identical to that used for the original definition. Only the dimension of the problem differs. Both cases involve verifying that a set of unilateral constraints prevents all motions of the system under investigation. In the original definition, the set of contact constraints was modeled to $1^{\text {st }}$ order by the expression:

$$
\boldsymbol{P} \dot{\boldsymbol{u}} \geq \mathbf{0}
$$

In the definition extended to the case of underactuated grasps (section III), all of the unilateral constraints are modeled to $1^{\text {st }}$ order by the expression:

$$
\boldsymbol{M} \dot{\boldsymbol{w}} \geq \mathbf{0}
$$

In both cases, the study of form-closure requires to solve a classical linear programming problem. This requires determining all solutions which satisfy a set of linear constraints. If this set is reduced to the null vector, then the grasp is form-closed. This analogy allows us to extend to underactuated grasps several relevant works that have previously been demonstrated for the original definition of form-closure. Therefore, this section aims to extend the method of form-closure analysis presented in section II.B, as well as the works on the minimum number of contact points necessary for $1^{\text {st }}$ order form-closure.

\section{A. A geometric method for form-closure analysis}

The geometric method described in this section is derived from [32]. It also requires the construction of the convex hull $\operatorname{conv}(\boldsymbol{M})$, formed by the lines of matrix $\boldsymbol{M}$, and then analyses the position of the origin of the configuration space of the grasp relatively to $\operatorname{conv}(\boldsymbol{M})$.

Hence, by analogy with the statements given in section II.B, the following propositions can be written:

(i) the grasp is not form-closed if the origin of the configuration space of the grasp is situated strictly outside $\operatorname{conv}(\boldsymbol{M})$, (ii) the grasp is $1^{\text {st }}$ order form-closed if, and only if, the origin of the configuration space of the grasp is situated strictly inside $\operatorname{conv}(\boldsymbol{M})$,

(iii) form-closure of the grasp is uncertain when the origin of the configuration space of the grasp is situated on the boundary of $\operatorname{conv}(\boldsymbol{M})$.

This work is illustrated with, once again, the examples of underactuated parallel-jaw gripper described in section II.C. The matrix $\boldsymbol{M}$, for the gripper shown in Fig. 3.c, is given by (41). In this case, the convex hull $\operatorname{conv}(\boldsymbol{M})$ does indeed contain the origin strictly in its interior (Fig. 10.a). The grasp is $1^{\text {st }}$ order form-closed.

In the same manner, the matrix $\boldsymbol{M}$ for the gripper shown in Fig. 3.d is given by (47). In this case, the origin is situated on the boundary of the convex hull $\operatorname{conv}(\boldsymbol{M})$ (Fig. 10.b).

Moreover, it can be noted that the convex hull is only formed from three points, which is not sufficient to contain the origin strictly in its interior. The grasp will not be $1^{\text {st }}$ order formclosed, but will possibly be higher order form-closed.

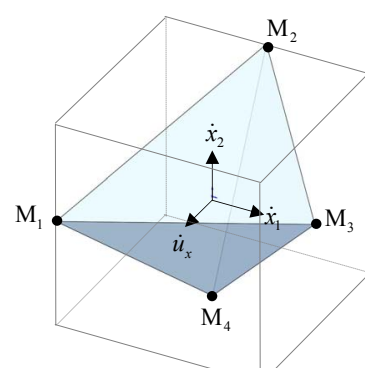

(a)

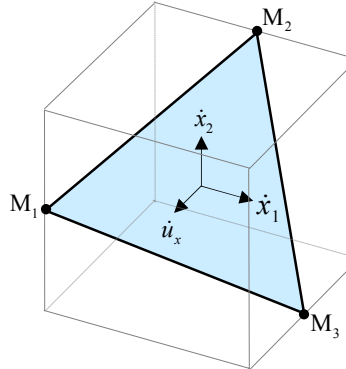

(b)
Fig. 10. Representation of the convex hull $\operatorname{conv}(\boldsymbol{M})$ for the underactuated gripper shown (a) in Fig. 3.c and (b) in Fig. 3.d.

\section{B. Minimum number of unilateral constraints for $1^{\text {st }}$ order form-closure}

This section aims to extend an important result, that of the minimum number of contact points necessary for $1^{\text {st }}$ order form-closure. These works were initiated by [32] and [37], and later demonstrated by [38], [39] and [33]. These works demonstrate that $d+1$ contacts are necessary for $1^{\text {st }}$ order form-closure:

$$
n_{c} \geq d+1
$$

where:

- $n_{c}$ is the number of unilateral contact constraints and

- $d$ is the dimension of the configuration space of the object (3 for the planar examples depicted in Fig. 2, 6 in the general case).

By analogy between propositions (8) and (37), the following inequality can be written:

$$
n_{k} \geq g+1
$$

where:

- $n_{k}$ is the number of unilateral constraints of the problem and 
- $g$ is the dimension of the grasp configuration space.

This can be stated the following way:

g+1 unilateral constraints are necessary for $1^{\text {st }}$ order formclosure, where $g$ is the dimension of the grasp configuration space.

The unilateral constraints of the problem are composed of the unilateral contact constraints and the unilateral constraints imposed by the unidirectional mechanisms (such as worm gears or free wheels). Hence:

$$
n_{k}=n_{u}+n_{c}
$$

with:

- $n_{u}$, the number of unilateral constraints imposed by the unidirectional mechanisms, and

- $n_{c}$, the number of unilateral contact constraints.

Additionally, the grasp configuration space is made of the configuration space of the object and the hand considered separately. Hence:

$$
g=n_{p}+d
$$

with:

- $n_{p}$, the dimension of the hand configuration space, that is, the number of dof of the hand, and

- $d$, the dimension of the configuration space of the object. Consequently, relation (51) can be rewritten as:

$$
n_{u}+n_{c} \geq n_{p}+d+1
$$

Inequality (54) enables a simple rule for designing underactuated hands, capable of performing $1^{\text {st }}$ order formclosed grasps:

When considering that each phalanx exerts one contact point with the object including the palm $\left(n_{c}=n_{p}+1\right)$, then

at least $d$ unidirectional mechanisms are required for the hand to be capable of performing $1^{\text {st }}$ order form-closed grasps.

On the basis of this new rule, let us review the existing underactuated hands described in section II.A. 2 and listed in Table II. It can be noted that, out of the 19 hands, only 4 integrate at least six unidirectional mechanisms: the Lopez hand, the Southampton hand, and the Barrett hand. The other hands cannot achieve $1^{\text {st }}$ order form-closed grasps of any object in space $(d=6)$. Of course, these hands are capable of performing stable grasps, exhibiting other types of stability such as higher order form-closure or force-closure.

One of the major limitations of underactuated hands lies in the ejection phenomenon described in [1]. As already mentioned, in certain configurations of the finger, the force exerted by certain phalanxes on the object must be negative to achieve static equilibrium of the finger. Because the contact forces are unilateral, this equilibrium cannot be attained, which causes a backward motion of the corresponding phalanxes. Introducing a unidirectional mechanism in the transmission of the closing motion of each phalanx prevents any backward motion of the phalanx and consequently eliminates the ejection phenomenon. The TWIX hand was designed according to this simple observation [40]. The underactuated hand of [20] also follows this simple design rule.

\section{PERSPECTIVES: \\ INTRODUCTION TO HIGHER ORDER FORM-CLOSURE OF UNDERACTUATED GRASPS}

As it has been shown in section III.B, the majority of underactuated hands does not use a sufficient number of unidirectional mechanisms to be able to achieve $1^{\text {st }}$ order form-closed grasps. However, some of these hands can achieve higher order form-closed grasps. In this section, a method is introduced for the analysis of higher order formclosure based on a $1^{\text {st }}$ order model of the grasp. Indeed, for sake of simplicity, $2^{\text {nd }}$ or higher order effects are not introduced in the model of the grasp.

In section III.B.3, a necessary condition for higher order form-closure of underactuated grasps, proposition (iii), has been expressed. Let $\boldsymbol{w}_{0}$ be the vector describing the grasp configuration that verifies this condition (meaning that at least one component $\dot{q}_{c, i}$ of the constraint vector is zero while the others are negative). To practically verify whether or not this grasp achieves higher order form-closure, it is proposed to span a discretized set of grasp configurations in the neighborhood of $\boldsymbol{w}_{0}$, obtained after an infinitesimal displacement $\boldsymbol{d} \boldsymbol{w}$ permitted by unilateral constraints. If for each neighbor configuration, the grasp tends to return to its original configuration $\boldsymbol{w}_{0}$, then the grasp is form-closed. This method also applies to the case of grasps where the contacts can be considered as fixed; in that case it is sufficient to study the matrix $\boldsymbol{P}$.

This method can be illustrated using the example shown in Fig. 2.b. As contacts are considered to be fixed in space, matrix $\boldsymbol{P}$, associated to vector $\dot{\boldsymbol{u}}=\left[\begin{array}{lll}\dot{u}_{x} & \dot{u}_{y} & \dot{\psi}_{z}\end{array}\right]$ representing object velocities in the plane, is directly studied:

$$
\boldsymbol{P}=\left[\begin{array}{ccc}
0 & 1 & 0 \\
-\sqrt{3} / 2 & -1 / 2 & 0 \\
\sqrt{3} / 2 & -1 / 2 & 0
\end{array}\right]
$$

The permissible velocities are given by the kernel of the matrix $\boldsymbol{P}$, the base of which is formed by the vector $\left[\begin{array}{lll}0 & 0 & 1\end{array}\right]^{T}$. Indeed, any vector $\dot{\boldsymbol{u}}$ belonging to the kernel of $\boldsymbol{P}$ is such that $\boldsymbol{P} \dot{\boldsymbol{u}}=\mathbf{0}$; the contact constraints are therefore respected whatever $\dot{\boldsymbol{u}} \in \mathcal{N}(\boldsymbol{P})$. In practice, this means that the object can rotate around the $z$ axis in both directions (Fig. 11.d).

Let us consider two new neighbor configurations to the original configuration, obtained respectively after an infinitesimal positive rotation and an infinitesimal negative rotation about $z$. The new matrix $\boldsymbol{P}$ is calculated for each neighbor configuration, after having modified the normals to the contacts. Then, the new space of permissible velocities (Fig. 12) can be deduced. It appears that after an infinitesimal 
negative rotation, the object in the new configuration can only rotate in the positive direction. In the same manner, after an infinitesimal positive rotation, the object in the new configuration can only rotate in the negative direction. The original configuration is therefore stable, since the object tends to return to it when displaced. It can be concluded that the grasp is form-closed (to an order higher than 1).

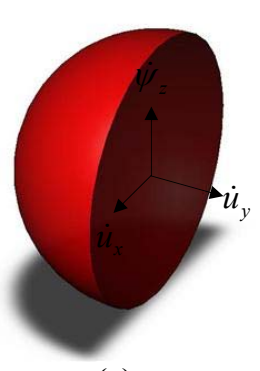

(a)

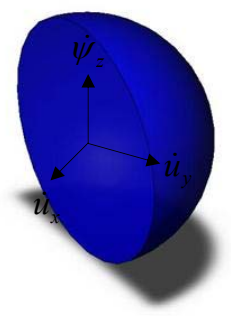

(c)

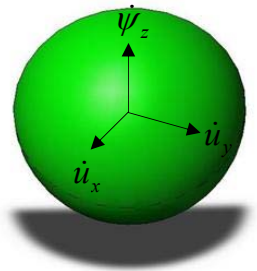

(b)

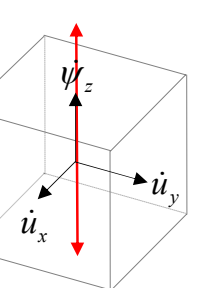

(d)
Fig. 11. (a) (b) (c) Representation of the half spaces forbidden by each of the three contacts for the grasp shown in Fig. 2(b). The union of the three open domains is represented in (d). It is a line which corresponds to the permissible velocities of the object in the original configuration $\boldsymbol{w}_{0}$.

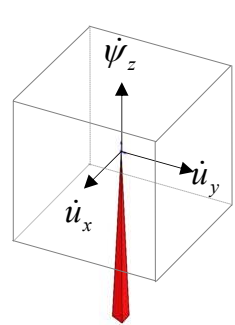

(a)

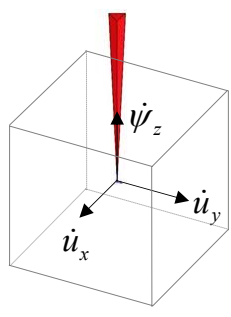

(b)
Fig. 12. Representation of the domains of permissible velocities after an infinitesimal positive rotation around $z$ (a), after an infinitesimal negative rotation around $z$ (b), for the grasp shown in Fig. 2(b). In both cases, the object tends to return to its original configuration $\boldsymbol{w}_{0}$.

Let us now consider the case of the underactuated paralleljaw gripper represented in Fig. 3.d, with the object in the centered position. The expression for the unilateral conditions is given in section III.C, from which the matrix $\boldsymbol{M}$ is deduced, with $\dot{\boldsymbol{w}}=\left[\begin{array}{lll}\dot{u}_{x} & \dot{x}_{1} & \dot{x}_{2}\end{array}\right]^{T}$ :

$$
\boldsymbol{M}=\left[\begin{array}{ccc}
1 & -1 & 0 \\
-1 & 0 & 1 \\
0 & \frac{\sin \left(\theta_{2}-\theta_{0}\right)}{\sin \left(\theta_{1}+\theta_{2}\right)} & -\frac{\sin \left(\theta_{1}+\theta_{0}\right)}{\sin \left(\theta_{1}+\theta_{2}\right)}
\end{array}\right]
$$

When the object is in the centered positioned, $u_{x}=0$, then $\theta_{0}=0$ and $\theta_{1}=\theta_{2}$. The matrix $\boldsymbol{M}$ is singular, the base of the kernel is given by the vector $\boldsymbol{e}_{1}=\left[\begin{array}{lll}1 & 1 & 1\end{array}\right]^{T}$. Hence, any motion of the grasp, $\dot{\boldsymbol{w}}$, such that $\dot{u}_{x}=\dot{x}_{1}=\dot{x}_{2}$ is permitted by the unilateral constraints (Fig. 13.a). Both neighbor configurations of the original configuration are studied; they are obtained after an infinitesimal displacement according to the vector $\boldsymbol{e}_{1}$ in the positive direction, $\dot{u}_{x}=\dot{x}_{1}=\dot{x}_{2} \geq 0$, and the negative direction. The matrix $\boldsymbol{M}$ is determined for both configurations, after having modified the values for the angles $\left(\theta_{0}, \theta_{1}, \theta_{2}\right)$. Then, the new domain of permissible velocities $\dot{\boldsymbol{w}}$ is deduced for each configuration. As in the previous example, it can be concluded that the grasp is form-closed because the grasp tends to regain its original configuration when it is displaced.

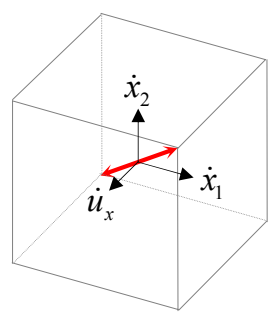

(a)

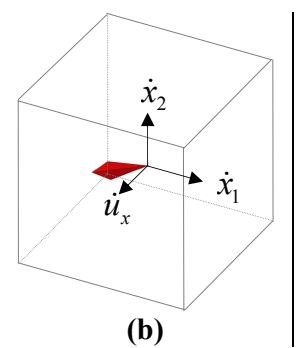

(b)

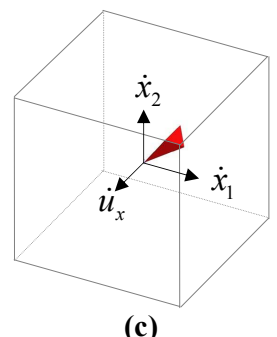

(c)
Fig. 13. Representation of the domain of permissible velocities for the gripper of Fig. 3.d, when the object is in the centered position (a); after an infinitesimal displacement of the grasp along the vector $\boldsymbol{e}_{1}$ belonging to the kernel of $\boldsymbol{M}$ in the positive direction $\dot{u}_{x}=\dot{x}_{1}=\dot{x}_{2} \geq 0$ (b); and in the negative direction (c). In both cases, the grasp tends to return to its original configuration.

It is this characteristic which distinguishes the gripper of Fig. 3.d from that of Fig. 3.c. Indeed, when the configuration of the grasp (c) changes, the grasp matrix $\boldsymbol{M}$ remains unchanged, which signifies that the object is not constrained to regain a stable equilibrium position. It can therefore be concluded that the grasp exerted by this gripper is not formclosed.

Both examples presented above can be solved graphically, because the dimension of the studied velocities space allows it. When the dimension is greater than three, it could be verified that, for each neighbor configuration of the original configuration, the infinitesimal displacement which needs to be realized by the grasp to return to the original configuration belongs to the permitted velocity space. Rigorously, this method does not constitute a formal proof of a higher order form-closure; rather it is a representation which allows us to go deeper in the understanding of this type of grasps.

\section{CONCLUSION}

In this paper, it has been pointed out that the definition of form-closure, as largely accepted in the literature, is inadequate when dealing with underactuated grasps. The definition is based on the assumption that contact points are fixed in space; this hypothesis is no longer true when the grasp 
is exerted by an underactuated hand. Therefore, a more general definition has been proposed, which consists in verifying that the system formed by the hand and the object is immobilized by a set of kinematic constraints. Among these constraints are the contact constraints to which the constraints imposed by unidirectional mechanisms have been added. Indeed, it has been shown, using several simple examples, that unilateral mechanisms have a relevant influence on the stability of the grasp. Hence, a new classification of underactuated hands has been proposed, based on the study of the expression of contact forces. Numerous existing underactuated hands use unilateral mechanisms, without justifying it. Contact constraints have been reformulated considering that phalanxes are possibly moving and a $1^{\text {st }}$ order expression has been proposed for the constraints imposed by unidirectional mechanisms.

A necessary and sufficient condition for $1^{\text {st }}$ order formclosure of underactuated grasps has been stated. The mathematical formalism adopted is identical to that for the original form-closure. Thus, relevant works from the literature dealing with the original definition of form-closure can be extended to the case of underactuated grasps. Indeed, a method for $1^{\text {st }}$ order form-closure analysis of underactuated grasps and a necessary and sufficient condition on the minimum number of unilateral constraints required for $1^{\text {st }}$ order form-closure of underactuated grasps have been proposed.

Finally, this study allowed us to state a simple design rule on the minimum number of unidirectional mechanisms to be introduced into the fingers closing motion transmission of an underactuated hand, so that it is capable of producing $1^{\text {st }}$ order form-closed grasps. Very few existing hands fulfill this condition. However, they are capable of achieving stable grasps which exhibit higher order form-closure or forceclosure. Therefore, some initial thoughts on higher order formclosure for underactuated grasps have been proposed, consisting in a necessary condition and a method of analysis based on a $1^{\text {st }}$ order model of the grasp.

\section{REFERENCES}

[1] T. Laliberté, L. Birglen and C. Gosselin, "Underactuation in robotic grasping hands," in Japanese Journal of Machine Intelligence and Robotic Control, Special Issue on Underactuated Robots, Vol. 4(3), pp. 77-87, 2002

[2] A. Bicchi, "On the closure properties of robotic grasping," in Int. J. of Robotics Research, Vol. 14(4), pp. 319-334, 1995.

[3] M. Luo, T. Mei, X. Wang and Y. Yu, "Grasp Characteristics of an Underactuated Robot hand," in Proc. of IEEE Int. Conf. on Robotics and Automation (ICRA'04), pp. 2236-2241, New Orleans, LA, USA, April 26-May 1st, 2004.

[4] L. Birglen, T. Laliberté and C. Gosselin, "Underactuated Robotic Hands," Springer-Verlag, ISBN: 978-3-540-77458-7, 2008.

[5] IFToMM website, URL: http://www.iftomm.3me.tudelft.nl/.

[6] J. Butterfass, M. Grebenstein, H. Liu and G. Hirzinger, "DLR-hand II next generation of a dextrous robot hand," in Proc. of IEEE Int. Conf. on Robotics and Automation (ICRA'01), pp. 109-120, Seoul, Korea, May 21-26, 2001.

[7] R.O. Ambrose, H. Aldridge, R.S. Askew, R.R. Burridge, W. Bluethmann, M. Diftler, C. Lovchik, D. Magruder and F. Rehnmark, "Robonaut: NASA's space humanoid," in IEEE Intelligent Systems, Vol. 15(4), pp. 57-63, 2000.
[8] J. Ueda, Y. Ishida, M. Kondo and T. Ogasawara T, "Development of the NAIST-hand with vision-based tactile fingertip sensor," in Proc. of IEEE Int. Conf. on Robotics and Automation (ICRA'05), pp. 2332-2337, Barcelona, Spain, April 18-22, 2005.

[9] K. Kaneko, K. Harada and F. Kanehiro, "Development of multi-fingered hand for life-size humanoid robots," in Proc. of IEEE Int. Conf. on Robotics and Automation (ICRA'07), pp. 913-920, Roma, Italy, April 10-14, 2007.

[10] S. Krut, "A force-isotropic underactuated finger," in Proc. of IEEE Int. Conf. on Robotics and Automation (ICRA'05), pp. 2314-2319, Barcelona, Spain, April 18-22, 2005

[11] W. Townsend, "The BarrettHand grasper - programmably flexible part handling and assembly," in Industrial Robot: An International Journal, Vol. 27(3), pp. 181-188, 2000.

[12] T. Laliberté and C. Gosselin, "Actuation system for highly underactuated gripping mechanism," US patent $\mathrm{n}^{\circ}$ US6.505.870, 2003.

[13] B. Massa, S. Roccella, M.C. Carrozza and P. Dario, "Design and development of an underactuated prosthetic hand," in Proc. of IEEE Int. Conf. on Robotics and Automation (ICRA'02), pp. 3374-3379, Washington, USA, May 11-15, 2002.

[14] L. Birglen and C. Gosselin, "Force analysis of connected differential mechanisms: application to grasping," in Int. J. of Robotics Research, Vol. 25(10), pp.1033-1046, 2006

[15] S. Hirose and Y. Umetani, "The development of soft gripper for the versatile robot hand," in Mechanism and Machine Theory, pp. 351-359, 1978.

[16] H. Champeau, "Conception et réalisation d'une main mécanique anthropomorphe en vue de son implantation sur le robot manus," M.S. report, Poitiers University of Sciences, France, 1991.

[17] H. De Visser and J. Herder2000, "Force directed design of a voluntary closing hand prosthesis," in Journal of Rehabilitation Research and Development, Vol. 37(3), pp. 261-271, 2000.

[18] N. Dechev, W.L. Cleghorn, and S. Naumann, S, "Multiple finger, passive adaptive grasp prosthetic hand," in Mechanism and Machine Theory, Vol. 36(10), pp. 1157-1173, 2001.

[19] C. Light and P. Chappell, "Development of a lightweight and adaptable multiple-axis hand prosthesis," in Medical Engineering and Physics, Vol. 22(10), pp. 679-684, 2000.

[20] G. Lopez, "Préhenseur anthropomorphe à serrage automatique," French patent $n^{\circ}$ FR2 $222.404,2001$

[21] M. Carrozza, C. Suppo, F. Sebastiani, B. Massa, F. Vecchi, R. Lazzarini, M. Cutkosky, and P. Dario, "The SPRING hand: development of a selfadaptive prosthesis for restoring natural grasping," in Autonomous Robots, Vol. 16(2), pp. 125-141, 2004.

[22] M. Carrozza, G. Cappiello, G. Stellin, F. Zaccone, F. Vecchi, S. Micera, and P. Dario, "A cosmetic prosthetic hand with tendon driven underactuated mechanism and compliant joints: ongoing research and preliminary results," in Proc. of IEEE Int. Conf. on Robotics and Automation (ICRA'05), pp. 2672-2677, Barcelona, Spain, April 18-22, 2005.

[23] S. Nasser, D. Rincon, M. Rodriguez, "Design of an anthropomorphic underactuated hand prosthesis with passive-adaptive grasping capabilities," in Florida Conf. on Recent Advances in Robotics and Robot Showcase, Miami, Florida, USA, May 25-26, 2006.

[24] C. Cipriani, F. Zaccone, G. Stellin, L. Beccai, G. Cappiello, M.C. Carrozza, and P. Dario, "Closed-loop controller for a bio-inspired multifingered underactuated prosthesis," in Proc. of IEEE Int. Conf. on Robotics and Automation (ICRA'06), pp. 2111-2116, Orlando, Florida, USA, May 15-19, 2006

[25] A.M. Dollar and R.D. Howe, "Simple, robust autonomous grasping in unstructured environments," in Proc. of IEEE Int. Conf. on Robotics and Automation (ICRA '07), pp. 4693-4700, Roma, Italy, April 10-14, 2007.

[26] D.J. Gow, "Prostheses with Mechanically Operable Digit Members," Touch Emas LTD., Int. patent WO2007063266 (A1), June 7th 2007.

[27] R. M. Crowder, "An anthropomorphic robotic end effector", in Robotics and Autonomous Systems, Vol. 7, Issue 4, pp. 253-268, November 1991.

[28] A. Bicchi and V. Kumar, "Robotic grasping and manipulation," in Ramsete: articulated and mobile robots for services and technology, Springer-Verlag. pp. 55-74, 2001.

[29] E. Rimon and J.W. Burdick, "On force and form closure for multiple finger grasps," in Proc. of IEEE Int. Conf. on Robotics and Automation (ICRA '96), pp. 1795-1800, Minneapolis, MN, USA, April 22-28, 1996.

[30] J. Cheong, "Immobilizing grasps for two- and three-dimensional objects," Ph.D. Thesis, Utrecht University, The Netherlands, 2006. 
[31] A. Van der Stappen, C. Wentink, and M. Overmars, "Computing immobilizing grasps of polygonal parts," in Int. J. of Robotics Research, Vol. 19(5), pp. 467-479, 2000

[32] F. Reulaux, "The kinematics of machinery," MacMillan, NY, 1976.

[33] B. Mishra, J.T. Schwartz, and M. Sharir, "On the existence and synthesis of multifinger positive grips," in Algorithmica, Vol. 2(1), pp. 541-558, 1987.

[34] J.K. Salisbury and J.J. Craig, "Articulated hands: force control and kinematic issues," in Int. J. of Robotics Research, Vol. 1(1), pp. 4-17, 1982.

[35] J. Ponce, S. Sullivan, A. Sudsang, J. Boissonnat, and J.P. Merlet, "On computing four-finger equilibrium and force-closure grasps of polyhedral objects," in Int. J. Robotics Research, Vol. 16(1), pp. 11-35, 1996.

[36] L. Birglen and C. Gosselin, "Kinetostatic analysis of underactuated fingers," in IEEE Trans. on Robotics and Automation, Vol. 20(2), pp 211-221, 2004

[37] P. Somov, "Uber Gebiete von Schraubengeschwindigkeiten eines starren Korpers bei verschiedener Zahl von Stutzflachen,", in Zeitricht fur Mathematik und Physik, Vol. 45, pp. 245-306, 1900.

[38] K. Lakshminarayana, "Mechanics of form closure," ASME Technical paper $78-D E T-32$, pp. 2-8, 1978

[39] X. Markenscoff, L. Ni and C.H. Papadimitriou, "The geometry of grasping", in Int. J. of Robotics Research, Vol. 9(1), pp. 61-74, 1990

[40] V. Bégoc, S. Krut, E. Dombre, C. Durand and F. Pierrot, "Mechanical design of a new pneumatically driven underactuated hand," in Proc. of IEEE Int. Conf. on Robotics and Automation (ICRA'07), pp. 927-933, Roma, Italy, 2007.

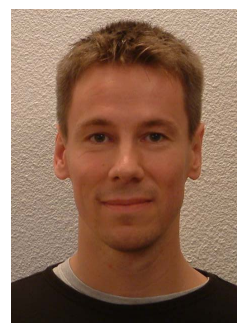

Sébastien Krut received the M.S. degree in mechanical engineering from the Pierre and Marie Curie University, Paris, France, in 2000 and the Ph.D. degree in automatic control from the Montpellier University of Sciences, Montpellier, France, in 2003. He has been a Post-doctoral fellow with the Joint Japanese-French Robotics Laboratory (JRL) in Tsukuba, Japan in 2004. He is currently a tenured research scientist in Robotics for the French National Centre for Scientific Research (CNRS), at the Montpellier Laboratory of Computer Science, Microelectronics and Robotics (LIRMM), Montpellier, France. His research interests include design and control of robotic systems.

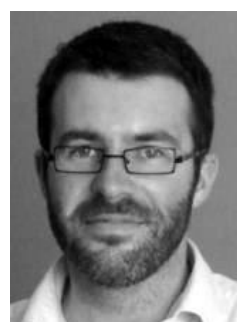

Vincent Bégoc received the M.Eng. degree in Mechanics and Automation from the "Institut Nationale des Sciences Appliquées", Rennes, France, in 2004. He received the Ph.D. degree in automatic control from the Montpellier University of Sciences, Montpellier, France, in 2008. He is currently working at $\mathrm{B}+$ Equipment, Gémenos, France, on robotic applications.

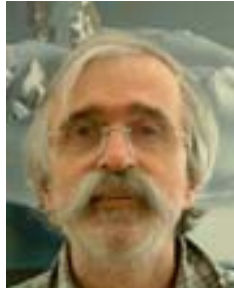

Etienne Dombre obtained his $\mathrm{PhD}$ degree in Automatic control in 1975 and his "Thèse d'Etat" in 1981, both from the Montpellier University of Sciences, Montpellier, France. He is a "Directeur de Recherche" for the French National Centre for Scientific Research (CNRS), currently at the Montpellier Laboratory of Computer Science, Microelectronics and Robotics (LIRMM), Montpellier, France. His research interests include mainly medical robotics.

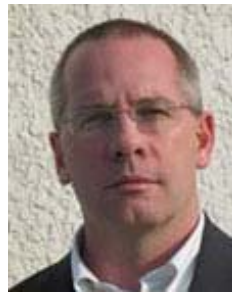

François Pierrot (M'93-SM'98) received the M.S. degree in mechanical engineering from the University of Paris, France, in 1986 and the Ph.D. degree in automatic control from the Montpellier University of Sciences, Montpellier, France, in 1991. He has been with the Montpellier Laboratory of Computer Science, Microelectronics and Robotics (LIRMM), since 1991, as a Researcher, Senior Researcher (1996) and "Directeur de Recherche" (2002) for the French National Centre for Scientific Research (CNRS). His research interests include mainly design and control of parallel manipulators. 